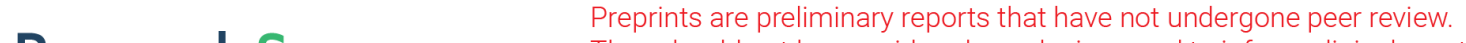 They should not be considered conclusive, used to inform clinical practice, or referenced by the media as validated information. \\ Detection Of Surface Defects And Subsurface Defects Of Polished Optics With Multisensor Image Fusion
}

Huanyu Sun

Zhejiang University

Shiling Wang

Zhejiang University

Xiaobo Hu

Zhejiang University

Hongjie Liu

China Academy of Engineering Physics

Xiaoyan Zhou

China Academy of Engineering Physics

Jin Huang

China Academy of Engineering Physics

Xinglei Cheng

Zhejiang University

Feng Sun

Zhejiang University

Yubo Liu

Zhejiang University

Dong Liu (D liudongopt@zju.edu.cn )

Zhejiang University https://orcid.org/0000-0002-2463-832X

\section{Research Article}

Keywords: Polished optics, Surface defects, Subsurface defects, Multisensor, Image fusion.

Posted Date: January 11th, 2022

DOI: https://doi.org/10.21203/rs.3.rs-1236950/v1

License: (9) (i) This work is licensed under a Creative Commons Attribution 4.0 International License.

Read Full License 


\title{
Detection of Surface Defects and Subsurface Defects of Polished Optics with Multisensor Image Fusion
}

\author{
Huanyu Sun ${ }^{1}$, Shiling Wang ${ }^{1}$, Xiaobo Hu${ }^{1}$, Hongjie Liu $^{2}$, Xiaoyan Zhou ${ }^{2}$, Jin Huang ${ }^{2}$, \\ Xinglei Cheng ${ }^{1}$, Feng $\operatorname{Sun}^{1}$, Yubo Liu ${ }^{1}$, Dong Liu ${ }^{1,3,4^{*}}$ \\ ${ }^{1}$ State Key Laboratory of Modern Optical instrumentation, College of Optical Science and Engineering: \\ International Research Center for Advanced Photonics, Zhejiang University. Hangzhou 310027. China \\ ${ }^{2}$ Research Center of Laser Fusion, China Academy of Engineering Physics, No.64 Mianshan Road, \\ 621000 Mianyang, Sichuan, China \\ ${ }^{3}$ Jiaxing Key Laboratory of Photonic Sensing \& Intelligent Imaging, Jiaxing 314000, China \\ ${ }^{4}$ Intelligent Optics \& Photonics Research Center, Jiaxing Research Institute Zhejiang University, \\ Jiaxing 314000, China \\ *Correspondence: liudongopt@zju.edu.cn
}

Abstract: Surface defects (SDs) and subsurface defects (SSDs) are the key factors decreasing the laser damage threshold of optics. Due to the spatially stacked structure, accurately detecting and distinguishing them has become a major challenge. Herein a detection method for SDs and SSDs with multisensor image fusion is proposed. The optics is illuminated by a laser under dark field condition, and the defects are excited to generate scattering and fluorescence lights, which are received by two image sensors in a wide-field microscope. With the modified algorithms of image registration and feature-level fusion, different types of defects are identified and extracted from the scattering and fluorescence images. Experiments show that two imaging modes can be realized simultaneously by multisensor image fusion, and HF etching verifies that SDs and SSDs of polished optics can be accurately distinguished. This method provides a more targeted reference for the evaluation and control of the defects of optics, and exhibits potential in the application of material surface research.

Keywords: Polished optics; Surface defects; Subsurface defects; Multisensor; Image fusion.

\section{Introduction}

Cutting, grinding and polishing are commonly used contact processing methods for optics, and they can cause surface defects (SDs) such as pits, scratches, and micro-cracks. These defects are not only distributed on the surface, but may further extend to a depth of several micrometers to hundreds of micrometers below the surface, becoming subsurface defects (SSDs). These defects will degrade the mechanical stability of the optics in extreme environment, such as space telescopes ${ }^{[1]}$ and deep- 
31 ultraviolet detectors ${ }^{[2]}$. And in high-power solid-state laser devices ${ }^{[3,4]}$, even small-sized defects can 32 cause the laser damage threshold to decrease, which becomes one of the key factors limiting the increase 33 of energy density ${ }^{[5,6]}$. It is necessary to detect and evaluate SDs and SSDs accurately, and reduce them 34 during processing or subsequent processing.

35 The detection of SDs is relatively mature at present. For example, the method using atomic force 36 microscope or electron microscope has high resolution, and is suitable for the detection in small 37 sampling areas. Efficient and fast detection of SDs of large optics can be realized by digital evaluation 38 system with wide-field scattering microscope ${ }^{[7,8]}$. But SSDs are covered under the surface, which are 39 difficult to directly detect by classic detection systems for SDs. Specific detection methods for SSDs 40 have been developed. Destructive ones such as acid etching ${ }^{[9]}$, dimpling ${ }^{[10]}$, etc. They expose SSDs 41 through physical or chemical means, and will cause irreversible damage to the optics. Non-destructive methods based on optical imaging include total internal reflection microscope (TIRM) ${ }^{[11]}$, confocal laser scanning microscope(CLSM $)^{[12,13]}$, and optical coherence tomography(OCT) ${ }^{[14]}$, etc. They illuminate the detection area in different ways, receive the optical signal modulated by SSDs, and will not cause damage. CLSM with fluorescence imaging is widely used for SSD detection in recent years ${ }^{[15,16]}$. The optics are doped with tiny fluorescent materials during grinding and polishing, and they can generate fluorescence under the excitation of lasers. These materials may come from the cooling fluid used in polishing ${ }^{[15]}$, or may be artificially doped quantum dots ${ }^{[17]}$. They are buried in the pits, scratches and other mechanical damage in subsurface layer. In addition, studies have shown that this kind of SSDs with fluorescence characteristics is closely related to the laser damage of optics ${ }^{[18,19]}$.

The surface of polished optics is relatively smooth, and most of the defects are removed, but there are still some residues randomly distributed on the surface and subsurface. Since SDs and SSDs may be stacked in space, it is a major challenge to quickly detect and accurately distinguish them by non54 destructive detection. CLSM with fluorescence imaging has capabilities of high resolution and three55 dimensional (3D) reconstruction. But the system is complex, and its field of view is quite small, which 56 is usually used for small-range detection of hundreds of microns. The large-range detection can be 57 realized by wide-field scattering imaging, but SDs and SSDs cannot be distinguished. Herein, a 
multisensor image fusion detection method is proposed, which combines wide-field scattering and

59 fluorescence imaging. The sample is illuminated by a laser, and a microscope system is placed in a

60 direction perpendicular to the sample. Scattering and fluorescence lights are split by the system, and received by two image sensors at the same time. Multisensor images are processed by spatial registration and feature-level fusion, realizing the identification and extraction of SDs and SSDs.

\section{Results and discussion}

\section{Multisensor imaging system}

65 Polishing is the last processing process of most optics, SDs and SSDs are greatly affected by polishing.

66 The surface and subsurface structure of a polished optics is shown in Fig. 1a. From top to bottom, there

67 are redeposition layer (or hydrolyzed layer, Beilby layer, polishing layer), subsurface layer, deformed

68 layer and the bulk ${ }^{[20]}$. According to the chemical action theory and thermal surface flow theory, the

69 redeposition layer is produced by the hydrolysis of the polishing slurry and the material surface.

70 Contaminants such as cerium and iron remain in this layer, and the thickness of this layer is about tens

71 to hundreds of nanometers. According to the mechanical grinding theory, polishing particles enter the

72 redeposition layer and then slide on the material, resulting in polishing dots and polishing scratches.

73 They and a small amount of cracks remaining during grinding together constitute mechanical SSDs.

74 Affected by the pulling force of polishing, contaminants are quickly buried in these SSDs, covering all

75 or part of SSDs under the redeposition layer. Therefore, there is SSD that extends below the surface and

76 is partially covered ${ }^{[21,22]}$. The former is called extended SSD and the latter is called covered SSD in this 77 paper.

78 The principle of dark-field scattering imaging for defects detection is shown in Fig. 1b. The surface 79 of the sample is illuminated by the incident light obliquely at a certain angle, and the light is directly 80 reflected when there are no defects on the surface. If there are defects, the scattering light is generated 81 and can be received by the microscopy system (not shown in the figure), so a dark-field image with 82 bright defects can be obtained. Since transmissive optics can be penetrated by the incident light, covered 83 SSDs and bulk defects ${ }^{[23]}$ will also be illuminated and generate scattering light, but the strength is 
relatively weak. Fluorescence imaging is similar to scattering imaging. As shown in Fig. 1c, the surface

85 is illuminated by the excitation light, then the fluorescence is generated by contaminants with strong light absorption. These contaminants are all over the surface and subsurface. The ones buried in SDs are removed after cleaning; and the ones buried in the redeposition layer and SSDs are retained and become the main source of fluorescence. Because of the deep mechanical SSDs, they provide places for a large number of contaminants to gather, so the fluorescence is strong and concentrated. The depth of the redeposition layer is very shallow, there are few contaminants and they are evenly distributed, so the

91 fluorescence from the redeposition layer is weak, showing as a uniform background.

(a)

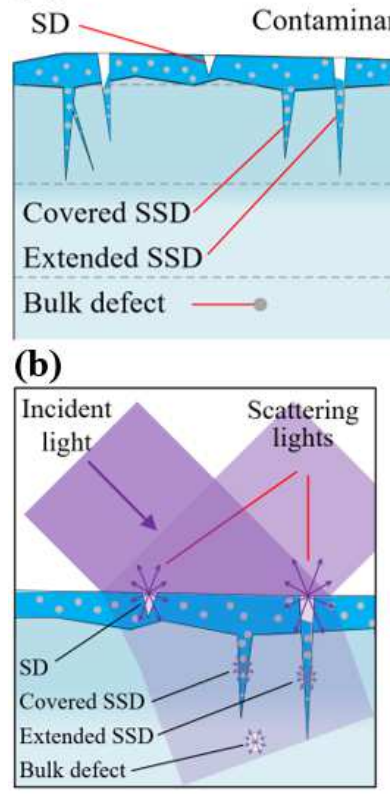

(d)

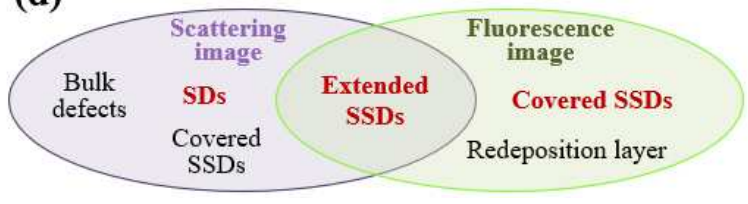

(c)

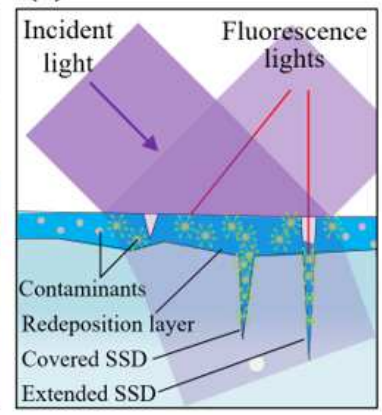

Extended SSD

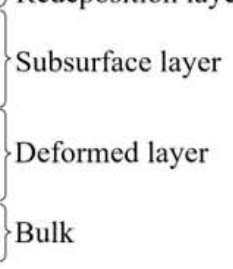

(e)

(e)
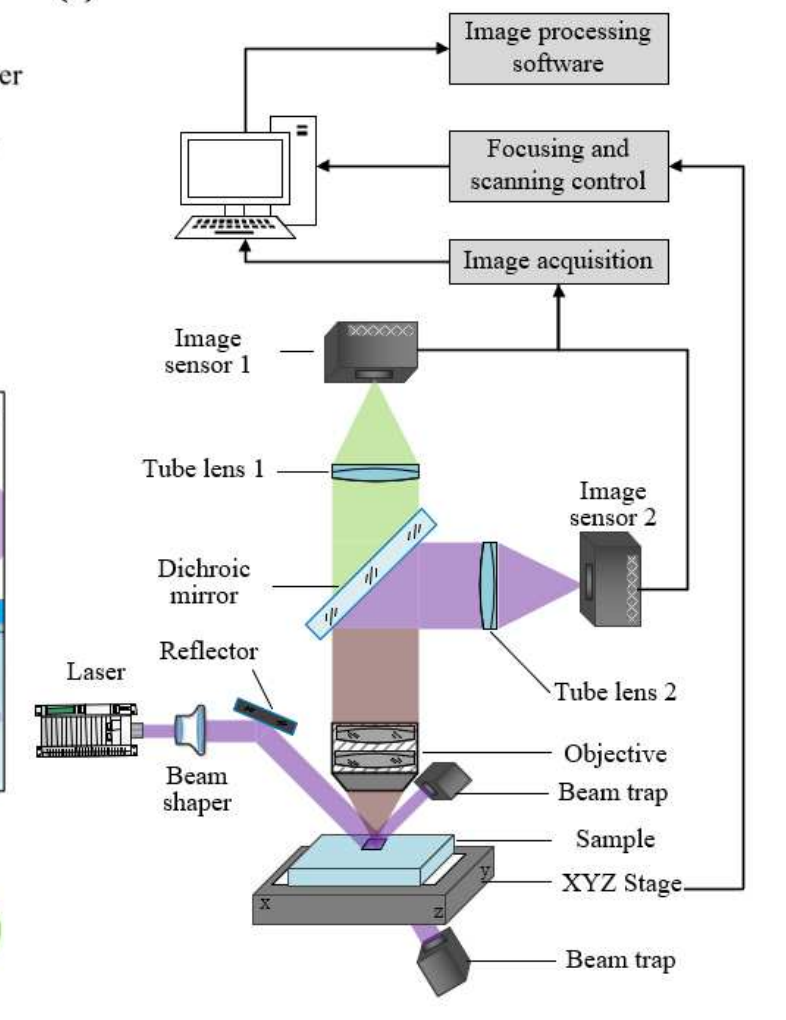

Fig. 1 Principle and layout of multisensor imaging system. a Surface and subsurface structure of

94 polished optics. b Scattering imaging principle of defects. $\mathbf{c}$ Fluorescence imaging principle of defects.

95 d Different types of defects characterized by scattering image and fluorescence image. e Layout of the 96 multisensor detection system.

As shown in Fig. 1d, SDs and extended SSDs are the main types of defects in scattering image,

and they are hard to distinguish. Extended SSDs and covered SSDs are the main types of defects in 
fluorescence image, and they are also hard to distinguish. The defects information collected by one

100 imaging method is very limited, so multisensor imaging includes both scattering and fluorescence

101 modes is proposed. Scattering and fluorescence lights are collected by two independent sensors

102 simultaneously to improve efficiency. Different types of defects are identified from the multisensor

103 images, so they can be reduced in a targeted manner in subsequent processing.

104 A detection system designed based on the principle of multisensor imaging is shown in Fig. 1e.

105 Two image sensors are used in the microscope to take scattering and fluorescence images simultaneously,

106 and a laser is used as the light source for two imaging modes. The sample used in the experiment is an

107 optical window of polished fused silica with a size of $100 \times 100 \mathrm{~mm}$ and a thickness of $5 \mathrm{~mm}$. When it is

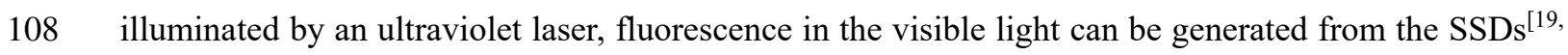

$109{ }^{24]}$. Therefore, a 355nm quasi-continuous laser is used as the excitation light in the system. The laser of

110 Gaussian beam is modulated into a uniform flat-top beam by a shaper, so the energy density of the

111 illuminated area is basically the same, covering the field of view of the imaging system. Reflected and

112 transmitted lights are absorbed by beam traps to reduce stray light. Scattering and fluorescence lights

113 generated by defects are received by an objective (4×, NA 0.13$)$, and after being split by a $409 \mathrm{~nm}$

114 dichroic mirror (transmission wavelength: $415-850 \mathrm{~nm}$ ), they enter different tube lenses ( $\mathrm{f}=150 \mathrm{~mm})$ and

115 image sensors respectively. The sensors are ultraviolet-enhanced CCD and electron-enhanced CCD

116 (pixel size: $13.3 \mu \mathrm{m}$, resolution: $1024 \times 1024$ ). Both sensors are located at a position conjugate to the

117 object plane, and take the scattering and fluorescence images on the same imaging area. The focusing

118 and scanning control system is used to control the 3D movement of the sample by a XYZ stage, and

119 adjusts the posture of the sample to keep it in focus positions.

120 Image processing includes preprocessing, registration and fusion. The scattering and fluorescence

121 images are first preprocessed after reading into the computer, including denoising, background

122 homogenization, and distortion correction. Then the defects will be highlighted from the background,

123 which is conducive to the subsequent image processing. Next, two images will be registered and fused

124 to get images characterizing SDs, extended SSDs and covered SSDs respectively. 
126 Image registration refers to the process of matching multiple images of the same scene to make their

127 features correspond. These images with overlapping regions may be taken at different times, different

128 conditions, or different sensors. Even if the same objective lens, tube lens and image sensors are used

129 in the multisensor imaging system, the positions of image planes will be slightly different due to the

130 difference in the detection wavelength. In addition, there will inevitably be differences in the positions

131 of the imaging devices in the optical path, especially the image sensors. These factors will cause the

132 difference of the imaging range of the target.

133 As shown in Fig. 2a.i \& 2a.ii, the imaging results of polishing scratches and polishing dots are 134 represented by straight lines and rectangles. These defects are extended SSDs, which exist in both 135 scattering and fluorescence images. Due to the difference between the two imaging systems, there are 136 differences in the position, size and rotation angle of the same defects in the two images. The 137 unregistered and registered superimposition images are shown in Fig. 2a.iii \& 2a.iv (the defects in the 138 scattering image and the fluorescence image are set to red and green respectively for display). If the two 139 images are not registered, the same defect cannot be overlapped in the superimposition image. In the 140 subsequent image fusion process, it is easy to misjudge the spatial location of such defects. Therefore, 141 image registration become one of the key steps before image fusion.

142 The flow chart of image registration is shown in Fig. 2b. First, the feature points are selected in the 143 scattering and fluorescence images respectively, and then the parameters of the transformation matrix 144 are estimated based on affine transformation. Finally, the image is resampled and interpolated to 145 complete the registration. After the system is set up, multisensor imaging system will no longer change, 146 so the affine transformation matrix is also fixed. Therefore, after the matrix is estimated for the first 147 time, the parameters can be used directly to complete image registration. 
(a)
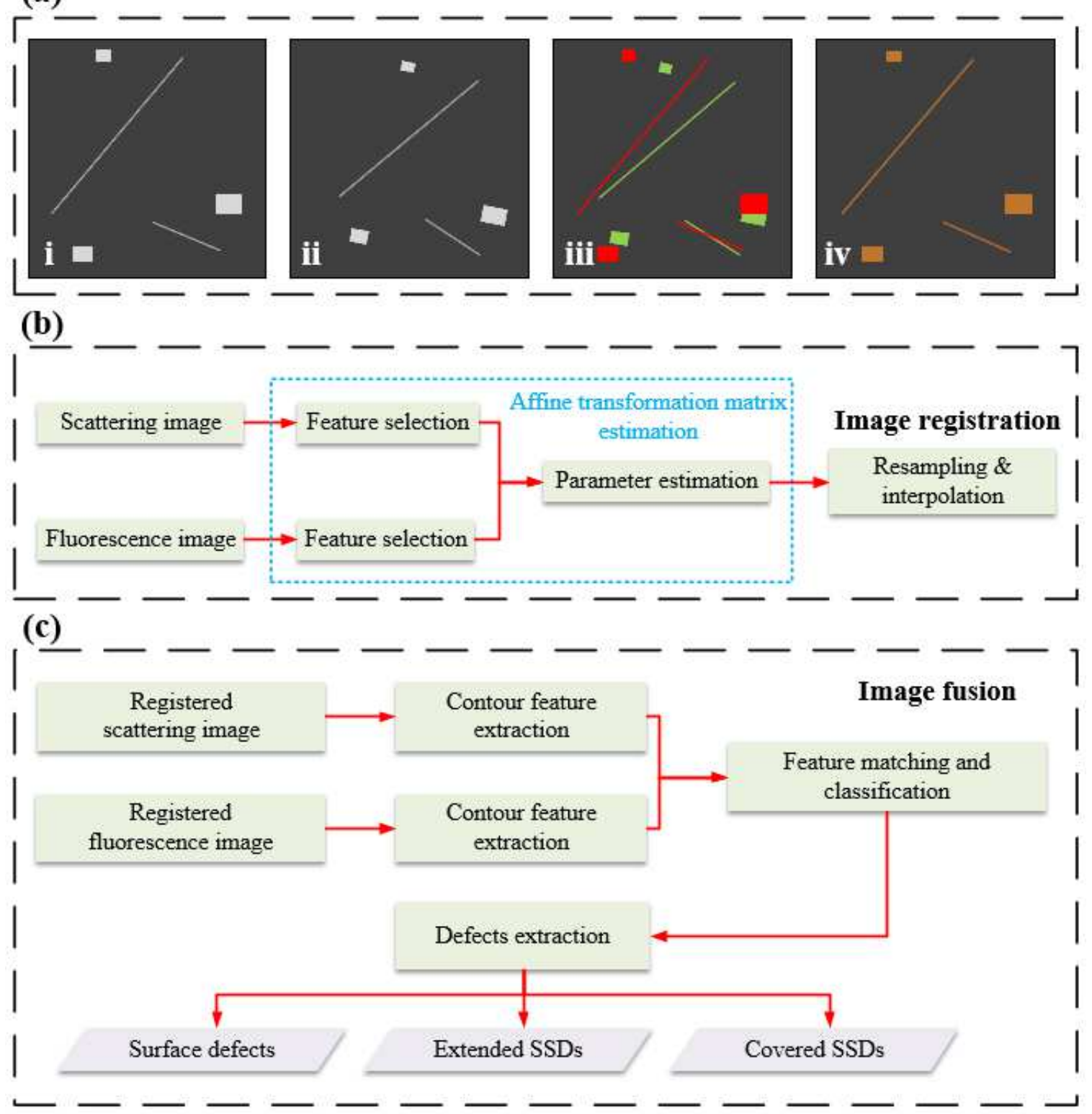

149 Fig. 2 Image registration and fusion. a Diagrams of image registration. i. scattering image; ii. 150 fluorescence image; iii. unregistered superposition image; iv. registered superposition image. b Flow 151 chart of image registration. $\mathbf{c}$ Flow chart of image fusion.

152 Image fusion is a multi-level image processing that uses the temporal or spatial correlation and 153 complementarity of two (or more) images to get more accurate fusion images. For the multisensor image

154 fusion detection system, the images taken by two sensors are based on different imaging principles,

155 having different physical meanings. The information of the scattering and fluorescence images is both 156 complementary and redundant, and the purpose of image fusion is to get images that characterize 
158 is used in this paper, and the flow chart is shown in Fig. 2c. First, the contours of all defects in the

159 scattering and fluorescence images are extracted, and the coordinates of the contour points are recorded.

160 Then the coordinates are used for feature matching, and the defects are divided into three types. Finally,

161 the defects are classified and extracted, getting images that characterize SDs, extended SSDs and 162 covered SSDs respectively.

\section{Imaging and identification of typical defects}

164 The imaging results of SDs and covered SSDs are shown in Fig. 3a-3f, they are superimposition images

165 of scattering and fluorescence images. The red areas in Fig. 3a-3b are surface scratches. They only exist

166 in the scattering image, indicating that they are SDs. The green areas in Fig. 3c-3d are polishing points,

167 and in Fig. 3e-3f are polishing scratches. These four defects are all covered SSDs, which are covered

168 under the redeposition layer and cannot generate obvious scattering lights, only existing in the

169 fluorescence image. The sample is etched with HF acid to verify the effectiveness of multisensor

170 imaging for SSDs detection. As shown in Fig. 3g-3h, the covered SSDs are fully exposed after etching,

171 which can be directly observed by a bright field microscope.

(a)

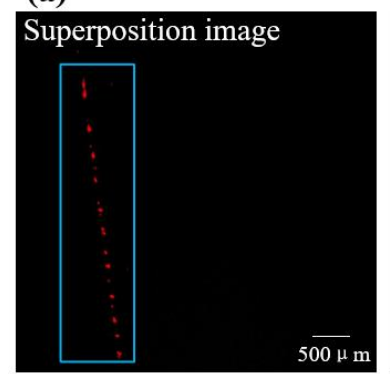

(e)

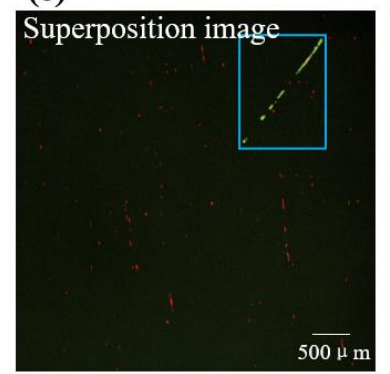

(b)

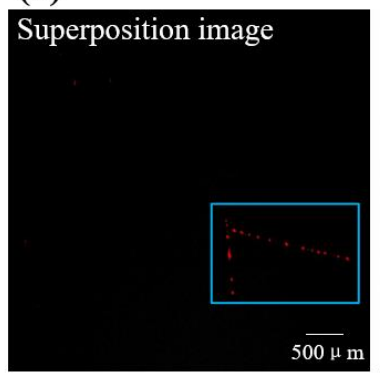

(f)

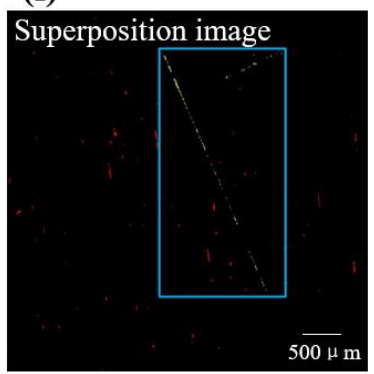

(c)

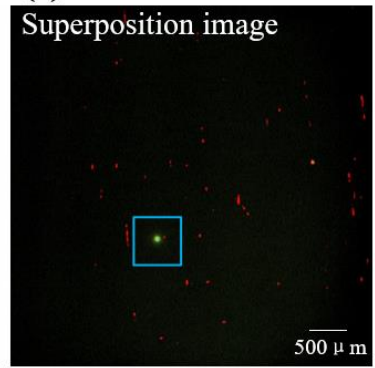

(g)

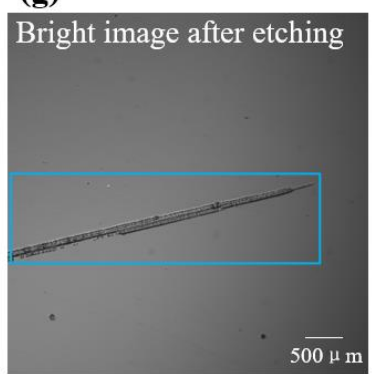

(d)

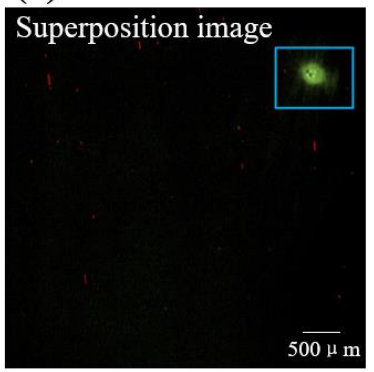

(h)

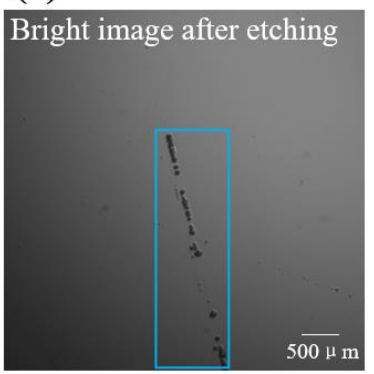

173 Fig. 3 Imaging results and etching verification of SDs and covered SSDs. a b Superposition images, 
174 there are SDs in the figures (red areas). c d e f Superposition images, there are covered SSDs in the 175 figures (green areas). $\mathbf{g}$ h Bright images, there are covered SSDs after etching.

176 The imaging result of an extended SSD is shown in Fig. 4a. This defect exists in both the scattering 177 and fluorescence images, and the overlapping area is displayed in yellow (red in the scattering image, 178 green in the fluorescence image, and the overlapping area becomes yellow after superimposition). To 179 get an image characterizes SDs, the extended SSD is needed to be removed from the scattering image.

180 The removal result after the pixel-level processing is shown in Fig. 4b. The defect in the fluorescent 181 image is first expanded, and then the expanded area is subtracted from the scattered image. It can be 182 seen from the result that the defect is not completely removed, but a "doughnut" remains, which is 183 physically meaningless. Although the "doughnut" can be removed by adjusting the expansion 184 parameters, but it is difficult to set parameters suitable for different sizes of defects. The removal result 185 after the feature-level image fusion is shown in Fig. 4c. It can be seen that the extended SSD is 186 completely removed, and the remaining defects in the figure are SDs.

(a)

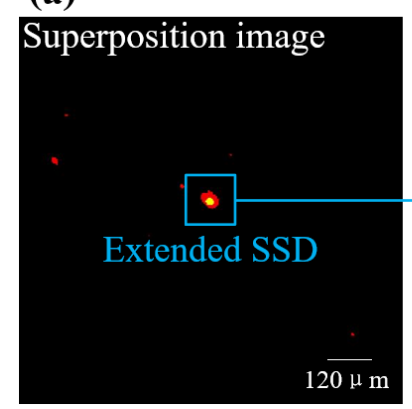

(b)

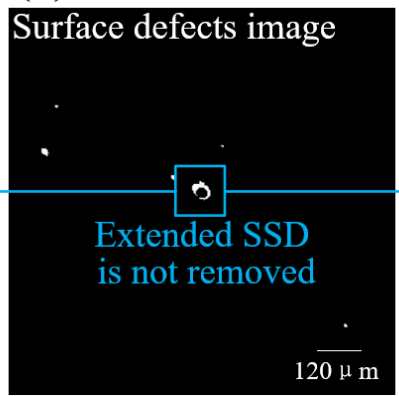

(c)

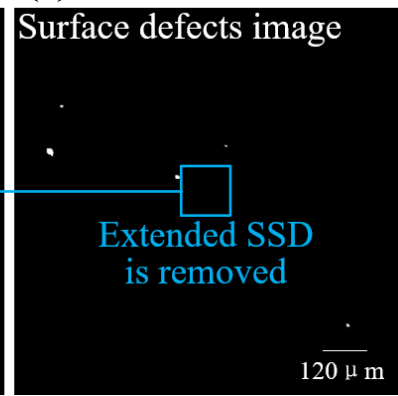

Fig. 4 Imaging and removal results of extended SSDs. a Superposition image. b SDs image (the extended SSD is not completely removed). c SDs image (the extended SSD is completely removed).

The superimposition images taken in three sites are shown in Fig. 5a - 5c. There are extended SSDs

191 in all three images, and image fusion is needed to extract different types of defects. There is a scratch

192 that exists in both the scattering and fluorescence images in Fig. 5a, and after it is removed from the

193 scattering image, a SDs image is obtained as shown in Fig. 5d. There is also a scratch that exists in both

194 the scattering and fluorescence images in Fig. 5b, and after it is extracted, an extended SSDs image is

195 obtained as shown in Fig. 5e. There are two polishing dots in both the scattering and fluorescence images

196 in Fig. 5c, and after they are removed from the fluorescence image, a covered SSDs image is obtained 
198 in the images can be identified, and the sizes of the defects can be calculated with calibration ${ }^{[25]}$, finally

199 realizing the quantitative evaluation of various defects.

(a)

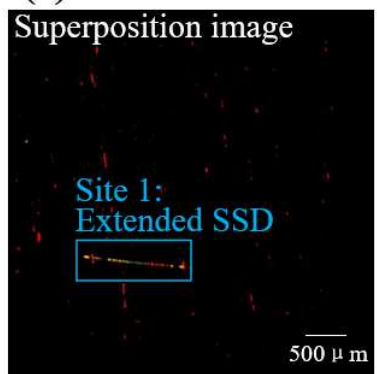

(d)

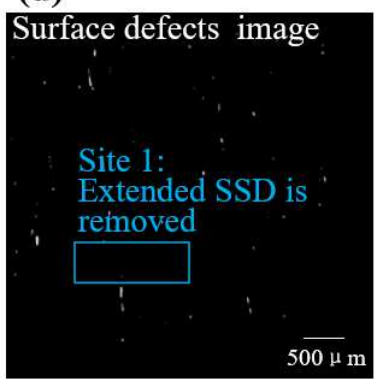

(b)

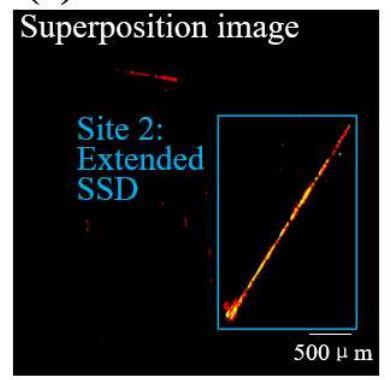

(e)

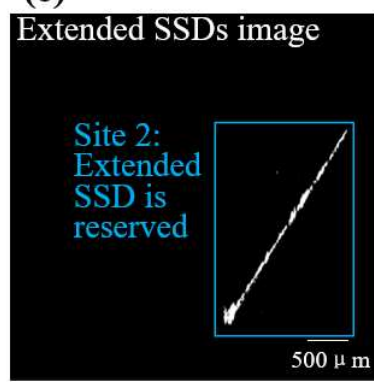

(c)

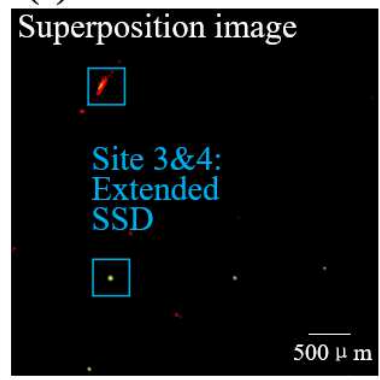

(f)

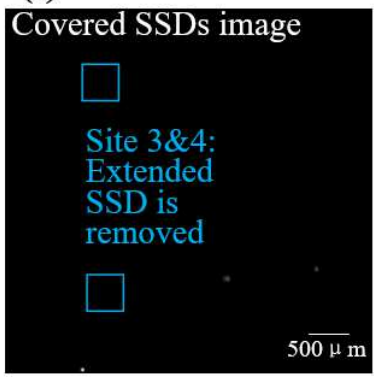

201 Fig. 5 Extraction results of three types of defects. a b c Superposition images of three sites. d Surface 202 detects image (the extended SSD in Fig. 5a is removed). e Extended SSDs image (the extended SSD in 203 Fig. 5b is reserved). f Covered SSDs image (the extended SSD in Fig. 5c is removed).

\section{Methods}

205 This section presents briefly the algorithms for image registration and fusion. In the beginning, 206 preprocessing is introduced. Whether it is a scattering image or a fluorescence image, the image pixels 207 where the defect is located have different gray-scale from the surrounding background, which is shown 208 as a bright spot on a dark background. However, the noise generated during image acquisition and the 209 uneven background generated by the illumination will adversely affect the extraction of defects. In the 210 previous paper, we have introduced the preprocessing algorithms such as Top-Hat algorithm, gray-scale 211 converting and medium filter ${ }^{[25]}$.

212 The core step of image registration, affine transformation matrix estimation is introduced next. For 213 two images to be registered for the same target, if the distance between a pair of feature points in the 
214 two images is small enough, it is considered that the points correspond to the same position in the target

215 object. This feature-based registration calculates the spatial transformation model between the images

216 by extracting the positional relationship between a pair of feature points. The analysis and processing

217 of the entire image is transformed into few points, which greatly reduces the amount of calculations. In

218 the multisensor image fusion detection system, the polished dots appear as bright spots under a dark

219 background in the scattering and fluorescence images, and the shapes are generally regular with obvious

220 closed area characteristics, which are suitable choices for feature points. The fluorescence and the

221 scattering image are used as fixed image and moving image respectively, and the feature points

222 coordinate sets of the fixed image $\left\{\left(x_{i}, y_{i}\right)\right\}$ and the moving image $\left\{\left(x_{i}^{\prime}, y_{i}^{\prime}\right)\right\}$ are established (i is the

223 number of selected feature points). As shown in Fig. 6a, three polishing dots that exist in both the

224 fluorescence and scattering images are selected as feature points. The centroid coordinates of the

225 polishing dots are used as feature point coordinates as Fig. $6 \mathrm{~b}$.

226 After the feature points are determined, the spatial transformation model parameters between the

227 fixed and moving images are calculated by affine transformation. The model of affine transformation

228 can be expressed as:

$$
\left[\begin{array}{l}
x \\
y \\
1
\end{array}\right]=\left[\begin{array}{ccc}
a_{1} & a_{2} & t_{x} \\
a_{3} & a_{4} & t_{y} \\
0 & 0 & 1
\end{array}\right]\left[\begin{array}{l}
x^{\prime} \\
y^{\prime} \\
1
\end{array}\right]=\mathbf{M}\left[\begin{array}{l}
x^{\prime} \\
y^{\prime} \\
1
\end{array}\right]
$$

230 where $(x, y)$ and $\left(x^{\prime}, y^{\prime}\right)$ are the pixel coordinates of the feature point pair in the fixed image and the 231 moving image respectively; $a_{1}, a_{2}, a_{3}, a_{4}$ are the transformation parameters of scale, rotation, flip 232 and shear; $t_{x}, t_{y}$ are the translation parameters. Substituting the coordinates $\left\{\left(x_{i}, y_{i}\right)\right\}$ and $233\left\{\left(x_{i}^{\prime}, y_{i}^{\prime}\right)\right\}$ into formula (1), the affine transformation matrix $\mathbf{M}$ can be calculated. Since there are 6 234 unknowns in formula (1), at least 3 pairs of non-collinear feature points are needed. The moving image 235 is resampled and interpolated according to the affine transformation matrix, and its coordinate system 236 is mapped to the coordinate system of the fixed image. 
(a)

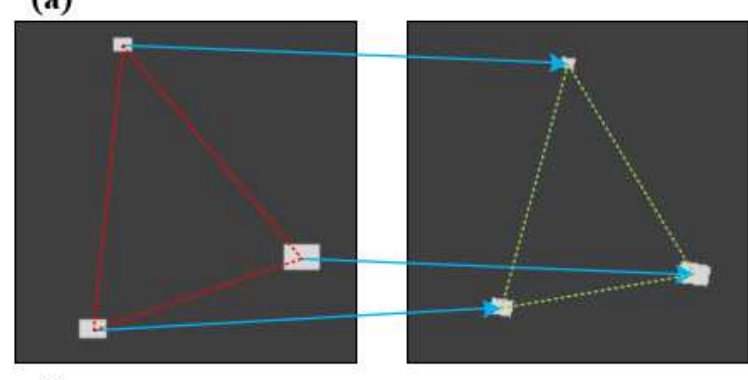

(c)

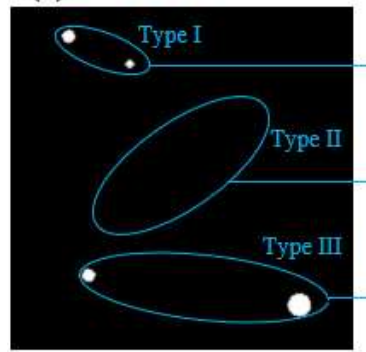

(e)
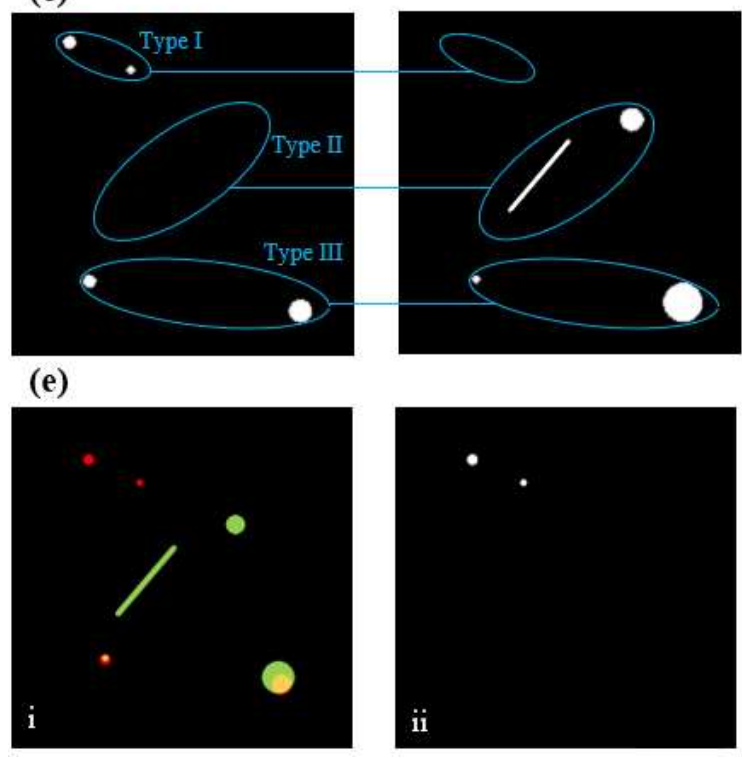

(b)

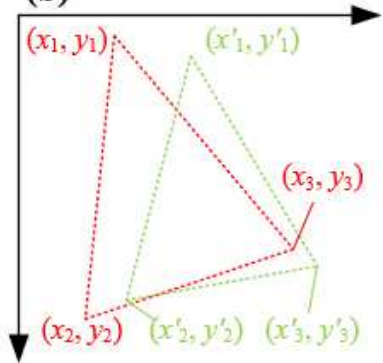

(d)

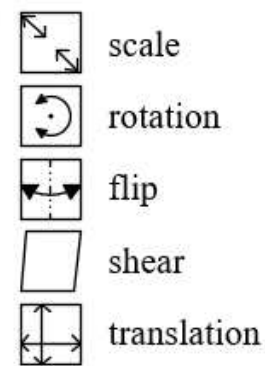

translation
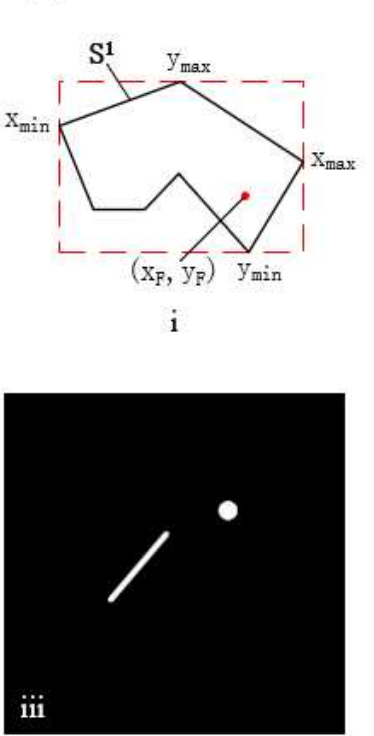
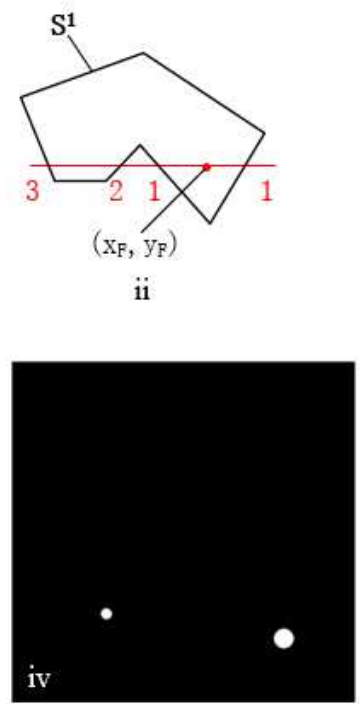

Fig. 6 Algorithm schematic diagrams for image registration and fusion. a Diagrams of feature points selection from two images to be registered (scattering image on the left, fluorescence image on the right).

240 b Diagrams of affine transformation model. c Simulation images of three types of defects (scattering 241 image on the left, fluorescence image on the right). d Diagrams of contour overlap judgment: i. coarse 242 judgment; ii. fine judgment. e Results of image fusion: i. superposition image; ii. type I defects (SDs); 243 iii. type II defects (covered SSDs); iv. Type III defects (extended SSDs).

244 The images are binarized after registration, and then they are processed by modified algorithm of 245 feature-level fusion. The algorithm consists of three steps: a. Contour feature extraction; $b$. Feature 246 matching and classification; c. Defects extraction. Next, they will be introduced in detail.

247 a. Contour feature extraction

248 The contour features in the image are extracted at first in the process of feature-level image fusion, 249 and a feature space is retained, reducing memory and time consumption. Traversal searching is used to 250 extract contour features of the defects, and the steps are as follows: 
i. First search the image from top to bottom, from left to right, and find the first white pixel as the contour point of the first defect, and record its coordinates as $\left(x_{1}, y_{1}\right)$.

253 ii. Take the first contour point as the center, and start the search for the second contour

iv. Repeat the above steps, establish the contour point coordinate set $F^{i}$ of all the defects in the figure, where $i$ is the number of the defect.

\section{The defects can be numbered while obtaining the contour point coordinates of each defect. If there} are a total of $m$ defects in the fluorescence image, the contour point coordinate set of the $i$-th defect is denoted as $F^{i}(i=1,2, \ldots, m)$; and there are a total of $n$ defects in the scattering image, the contour point coordinate set of the $j$-th defect is denoted as $S^{j}(j=1,2, \ldots, n)$.

267 b. Feature matching and classification

268 The commonly used feature matching method is template matching. If the morphological similarity 269 of the features in two images is greater than a threshold, the two features are considered to belong to the 270 same target. The defects are mainly linear scratches and dotted polished dots, and there will be a lot of 271 similar features in the image. And the scattering image and the fluorescence image are taken by different 272 imaging mechanisms, even the same defect may show different topography in the two images. Therefore, 273 template matching is not suitable for the feature matching of defects. The spatial information of the 274 defects is used for feature matching in this paper. As shown in Fig. 6c, if the contour of a defect in the 
275 scattering image does not overlap with any contour in the fluorescence image, the defect belongs to

276 Type I (SD, which only exists in the scattering image). If the contour of a defect in the fluorescence

277 image does not overlap with any contour in the scattering image, the defect belongs to Type II (covered

278 SSD, which only exists in the fluorescence image). If the contour of a defect overlaps on the two images,

279 the defect belongs to Type III (extended SSD, which exist in both scattering and fluorescence images).

280 The key to feature matching is to accurately determine whether the contour overlaps in the two

281 images. The judgment criterion used in this paper is: if there are two points on the contour of a defect

282 that are located within the area contained by the contour of another defect, the two defects are spatially

283 overlapped. For example, take one point $\left(x_{F}, y_{F}\right)$ in the first defect $F^{1}$ to determine whether it

284 locates within the irregular area contained by the first defect $S^{1}$. As shown in Fig. 6d.i, the rough

285 judgment is carried out first. The maximum and minimum values of the abscissa and ordinate in $S^{1}$ are

286 denoted as $x_{\max }, x_{\min }, y_{\max }, y_{\min }$ respectively. According to the coordinates of the above four points,

287 a circumscribed rectangle of $S^{1}$ can be constructed. If $\left(x_{F}, y_{F}\right)$ is outside this rectangle, that is:

$$
\left(x_{F}>x_{\text {max }}\right) \vee\left(x_{F}<x_{\text {min }}\right) \vee\left(y_{F}>y_{\text {max }}\right) \vee\left(y_{F}<y_{\text {min }}\right) .
$$

289 Then $\left(x_{F}, y_{F}\right)$ must be outside the irregular area of $S^{1}$, and the coarse judgment is completed. On the

290 contrary, if $\left(x_{F}, y_{F}\right)$ is within the circumscribed rectangle, the Ray Casting Algorithm ${ }^{[26]}$ is used for

291 fine judgment. As shown in Fig. 6d.ii, a horizontal straight line through $\left(x_{F}, y_{F}\right)$ is drawn, and the

292 number of times that the straight line intersects the contour of $S^{1}$ are calculated. If the number of 293 intersection points on the left and right sides of the point are both odd, then the point is inside the contour, 294 otherwise it is outside. The number of intersections on the left and right sides are 3 and 1 respectively 295 shown in Fig. 6d ii, so it can be judged that $\left(x_{F}, y_{F}\right)$ is in the irregular area contained by $S^{1}$.

296 The implementation of fine judgment are provide in references ${ }^{[26,27]}$. Compared with the point-by297 point comparison in the connected domain, this feature-level matching only traverses the points on the 298 contour, reducing the time required for feature matching. And the number of judgments is greatly 
reduced by two rounds of judgment. After all the points in $F^{1}$ are traversed, if there are two points

300 located in the irregular area contained by $S^{1}$, it means that the two defects $F^{1}$ and $S^{1}$ are overlapped.

301 Comparing all the features in $F^{i}$ and $S^{j}$, all the overlapped defects can be indentified and marked,

302 which belong to type III. After the marked defects removed from $S^{j}$, the remaining ones belong to

303 Type I. And after the marked defects removed from $F^{i}$, the remaining ones belong to Type II.

304 c. Defects extraction

305 After the classification of all defects is completed, the final step of image fusion is to extract defects

306 to get fused images with accurate physical meaning. The image in Fig. 6e.i is the superposition result

307 of the scattering and fluorescence images in Fig.6c. This image retains the information taken by two

308 sensors to the greatest extent, and Type I, Type II and Type III defects are set to red, green and yellow

309 respectively. Fig. 6e.ii - 6e.iv are fusion images after defects extraction. A single image does not cover

310 all the information taken by two sensors, but each one characterizes a type of defect and has a more

311 accurate physical meaning.

\section{Conclusion}

313 SDs can be detected by scattering imaging, but the detection results will be interfered by SSDs; SSDs

314 can be detected by fluorescence imaging, but it is difficult to distinguish between the extended SSDs

315 and covered SSDs. Based on the scattering and fluorescence imaging principles of polished optics, a

316 multisensor image fusion detection method for SDs and SSDs is proposed. Two image sensors are used

317 for wide-field imaging in two modes at the same time, which has the advantages of large imaging range

318 and high detection efficiency. The scattering and fluorescence images are processed by registration and

319 fusion algorithms, and after contour extraction, feature matching and classification, three types of

320 defects are extracted. The method provides a rich reference for the quality evaluation of the optical

321 surface processing, which is beneficial to improve the processing technology, reducing various defects

322 in a more targeted manner. 
Abbreviations

SDs: Surface defects; SSDs: subsurface defects (SSDs); TIRM: total internal reflection microscope; CLSM: confocal laser scanning microscope; OCT: optical coherence tomography; 3D: threedimensional; CCD: charge coupled device; HF: hydrogen fluoride.

\section{Acknowledgements}

The authors would like to express their great appreciation to Mr. An Lu and Mr. Menghui Huang for their helps in programming.

\section{Authors' contributions}

Huanyu Sun: Conceptualization, Investigation, Writing original draft. Shiling Wang: Conceptualization, Methodology. Xiaobo Hu: Software, Formal analysis. Hongjie Liu: Methodology. Xiaoyan Zhou: Resources. Jin Huang: Resources. Xinglei Cheng: Investigation. Feng Sun: Software. Yubo Liu: Software. Dong Liu: Supervision, Writing - review \& editing.

\section{Funding}

This work was supported by the National Key Research and Development Program of China (2016YFC1400900); National Natural Science Foundation of China (NSFC) (41775023); Excellent Young Scientist Program of Zhejiang Provincial Natural Science Foundation of China (LR19D050001); Fundamental Research Funds for the Central Universities(2019FZJD011); State Key Laboratory of Modern Optical Instrumentation Innovation Program (MOI2018ZD01).

\section{Availability of data and materials}

The calculation and experiment data that support the works of this study are available from the corresponding authors on reasonable request.

\section{Competing interests}

The authors declare that they have no competing interests.

\section{References}

[1] Serjeant S, Elvis M, Tinetti G. The future of astronomy with small satellites. Nat. Astron. 2020;4:1031-1038.

[2] Li Y, Zheng W, Huang F. All-silicon photovoltaic detectors with deep ultraviolet selectivity. PhotoniX. 2020;1:15.

[3] Betti R, Hurricane OA. Inertial-confinement fusion with lasers. Nat. Phys. 2016;12:435448.

[4] Zhang F, Cai HB, Zhou WM, Dai ZS, Shan LQ, Xu H,et al. Enhanced energy coupling for 
indirect-drive fast-ignition fusion targets. Nat. Phys. 2020;16:810-814.

[5] Demos SG, Staggs M, Kozlowski MR. Investigation of processes leading to damage growth in optical materials for large-aperture lasers. Appl. Opt. 2002;41:3628-3633.

[6] Neauport J, Lamaignere L, Bercegol H, Pilon F, Birolleau JC. Polishing-induced contamination of fused silica optics and laser induced damage density at $351 \mathrm{~nm}$. Opt. Express. 2005;13:10163-10171.

[7] Liu D, Wang S, Cao P, Li L, Cheng Z, Gao X,et al. Dark-field microscopic image stitching method for surface defects evaluation of large fine optics. Opt. Express. 2013;21:59745987.

[8] Zhang Y, Yang Y, Li C, Wu F, Chai H, Yan K,et al. Defects evaluation system for spherical optical surfaces based on microscopic scattering dark-field imaging method. Appl. Opt. 2016;55:6162.

[9] Neauport J, Ambard C, Cormont P, Darbois N, Destribats J, Luitot C, et al. Subsurface damage measurement of ground fused silica parts by HF etching techniques. Opt. Express. 2009; 17:20448-20456.

[10] Zhou Y, Funkenbusch PD, Quesnel DJ, Golini D, Lindquist A. Effect of Etching and Imaging Mode on the Measurement of Subsurface Damage in Microground Optical Glasses. J. Am. Ceram. Soc. 1994;77:3277-3280.

[11] Kozlowski MR. Application of total internal reflection microscopy for laser damage studies on fused silica. Proceedings of SPIE - The International Society for Optical Engineering. 1998;3244:282-295.

[12] Wang C, Tian A, Wang H, Li B, Jiang Z. Optical subsurface damage evaluation using LSCT. Proceedings of SPIE - The International Society for Optical Engineering. 2009;7522:

[13] Liu J, Liu J, Liu C, Wang Y. 3D dark-field confocal microscopy for subsurface defects detection. Opt Lett. 2020;45:660-663.

[14] Sergeeva M, Khrenikov K, Hellmuth T, Boerret R. Sub surface damage measurements based on short coherent interferometry. J. Eur. Opt. Soc-Rapid. 2010;5:138-138.

[15] Neauport J, Cormont P, Legros P, Ambard C, Destribats J. Imaging subsurface damage of grinded fused silica optics by confocal fluorescence microscopy. Opt. Express. 2009; 17:3543.

[16] Sun H, Wang S, Bai J, Zhang J, Huang J, Zhou X,et al. Confocal laser scanning and 3D reconstruction methods for the subsurface damage of polished optics. Opt. Lasers Eng. 2021;136:106315.

[17] Williams WB, Mullany BA, Parker WC, Moyer PJ, Randles MH. Using quantum dots to tag subsurface damage in lapped and polished glass samples. Appl. Opt. 2009;48:51555163.

[18] Liu H, Huang J, Wang F, Zhou X, Jiang X, Wu W,et al. Photoluminescence defects on subsurface layer of fused silica and its effects on laser damage performance. Proceedings of SPIE - The International Society for Optical Engineering. 2015;9255:

[19] Fournier J, Neauport J, Grua P, Fargin E, Jubera V, Talaga D, et al. Green luminescence in silica glass: A possible indicator of subsurface fracture. Applied Physics Letters. 2012;100:56.

[20] Suratwala TI, Miller PE, Bude JD, Steele WA, Shen N, Monticelli MV,et al. HF - Based Etching Processes for Improving Laser Damage Resistance of Fused Silica Optical Surfaces. J. Am. Ceram. Soc. 2011;94:416-428.

[21] Cook LM. Chemical processes in glass polishing. J. Non· Cryst. Solids. 1990;120:0-171.

[22] Rodolphe C, Jérôme N, Philippe L, Daniel T, Thomas C, Philippe C,et al. Using STED and ELSM confocal microscopy for a better knowledge of fused silica polished glass interface. Opt. Express. 2013;21:29769-29779.

[23] Wang S, Sun H, Liu A, Zhang J, Cheng X, Huang J,et al. Automatic evaluation system for bulk defects in optics. Opt. Lasers Eng. 2021;137: 
405

406

407

408

409

410

411

412

413

414
[24] Liu H, Wang F, Geng F, Zhou X, Huang J, Ye X,et al. Nondestructive detection of optics subsurface defects by fluorescence image technique. Optics and Precision Engineering. 2020;28:50-59.

[25] Liu D, Yang YY, Wang L, Zhuo YM, Lu CH, Yang LM,et al. Microscopic scattering imaging measurement and digital evaluation system of defects for fine optical surface. Opt. Commun. 2007;278:240-246.

[26] Heckbert PS. Graphics Gems IV. 4th ed. San Diego:Academic Press; 1994.

[27] Milgram M. Does a point lie inside a polygon? Journal of Computational Physics. 1989;84:134-144. 